\title{
The Applications and Thinking of "Micro-Teaching-Assistant" in the Teaching of Medical Physics
}

\author{
Wenbin Kang ${ }^{1,3, *}$, Baiping Liu ${ }^{2}$, Pengcheng Zhang ${ }^{1,3}$, Benchao Zhu ${ }^{1,3}$,Guangxiao He ${ }^{1,3}$ \\ and Jia Guo 1,3 \\ ${ }^{1}$ School of Public Health and Management, Hubei University of Medicine, Hubei Shiyan 442000, \\ China; \\ ${ }^{2}$ Dongfeng Senior High School, Shiyan 442008, China; \\ ${ }^{3}$ Bio-X research center, Hubei University of Medicine, Hubei Shiyan 442000, China. \\ wbkang@hbmu.edu.cn \\ *The corresponding author
}

Keyword: Micro-Teaching-Assistant; Medical Physics; Teaching;Practice; Application

\begin{abstract}
At present, the "micro-teaching-assistant" (MTA) has been widely applied and recognized by the higher education sector. Based on the classroom teaching practice of medical physics with MTA in Hubei University of Medicine, this paper talks about the application and reflection of MTA in medical physics teaching. Our teaching practice shows that the MTA is very popular with teachers and students, simple and convenient operation, efficient information dissemination. MTA significantly arouse the student's interest in learning and participation, significantly improve the academic performance. The research shows that it is beneficial to the promotion of MTA and the application in medical physics teaching, which is beneficial to the improvement of students' ability of autonomous learning, analyzing, and solving problems, which is conducive to the cultivation of scientific quality and innovation ability.
\end{abstract}

\section{“微助教”在医学物理学教学中的应用与

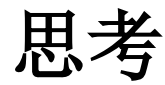

康文斌 $^{1,3}$, 刘百萍 ${ }^{2}$, 张鹏程 ${ }^{1,3}$, 朱本超 ${ }^{1,3}$ ，何光晓 ${ }^{1,3}$, 郭佳 ${ }^{1,3}$

1 湖北医药学院，公共卫生与管理学院，湖北十堰 442000

2 东风高级中学，湖北十堰 442001

3 湖北医药学院, Bio-X研究中心, 湖北十堰 442000

摘要：目前 “微助教” 得到了高等教育界的广泛应用和认可。本文基于湖北医药学院医学物理学 “微 助教” 课堂教学实践，谈谈 “微助教” 在医学物理学教学中的应用和反思。我们的教学实践表明，“微助 教” 很受师生的欢迎，操作简单方便，信息传播高效快捷，显著地提高了学生的学习兴趣和参与度，明 显地提高了学习成绩。研究表明，有利于 “微助教” 的推广及在医学物理学教学中的应用，有利于医学 生自主学习能力和分析解决问题能力的提高, 有利于科学素质和创新能力的培养。 
关键词: 微助教; 医学物理学; 教学; 实践; 应用

中图分类号: G40-057

文献标识码: A

\section{1 引言}

“微助教” 是 2016 年上线的一款新型的智慧校园课堂教学系统, 由华中师范大学心 理学院教师田媛和华中科技大学专业团队开发的微信公众平台。“微助教” 则是一款课堂 互动轻松应用工具, 它强调的是操作简便、方便实用、有趣味性的过程性评价和教学。它 能够为教师带来 “控制感”, 给学生带来 “参与感”, 给教育管理者带来 “安全感”。“微助 教” 提供课堂签到、课堂测试、课堂讨论等多种互动功能, 以游戏化思维鼓励学生积极参 与课堂互动, 以便捷操作鼓励教师积极开展教学实践与创新, 化繁为简, 对症下药, 提高 教学效率。通过 “微助教”, 学生可以用手机在课堂中签到、答题和讨论、出勤率、课堂 研讨、虚拟论坛发言、平时作业和小测验等都可以记录下来, 便于老师对学生学习的全过 程进行持续观察，做出最后发展性的评价。自 2016 年 4 月推广以来, “微助教” 凭借其简 便、高效、趣味性强等优势, 吸引了全国 20 多个省 700 余所高校 6000 余名教师在平台上 开设 6000 门课程, 开展课堂互动 90 万余人, 开展课堂互动 600 多万人次。笔者在湖北医 药学院医学物理学的教学中应用了 “微助教”, 这里将总结一下 “微助教” 的功能特点及 其在医学物理学教学应用中的思考。

\section{2 “微助教” 在医学物理学教学中的应用}

\section{1 在医学物理学中的应用}

\section{1.1 精准的 GPS 定位随时签到系统}

精准的 GPS 定位签到和随时签到系统给予了教师高度 “控制感”, 让学生不敢随意逃 课、旷课、迟到和早退等。

例如, 我们对某一班一学期的随机签到情况做了统计分析, 结果如图 1 所示。研究数 据表明, 首次使用 “微助教” 签到时, 只有 $75 \%$ 的出勤率, 后面几次发现出勤率维持较高 的状态。这充分说明了, “微助教” 在教师教学过程中, 方便了考勤和提高了学生出勤率。

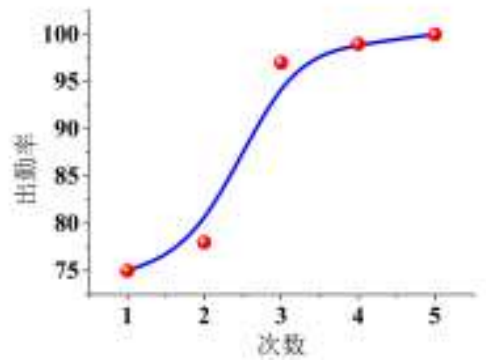


图 1 签到次数与出勤率关系曲线：横轴表示签到的次数; 纵轴表示签到出勤率

\section{1.2 及时的课堂答题互动}

作为一款课堂教学辅助工具, 微助教为教师提供了在线一站式出题、看题和查看答题 数据的服务, 加入课堂学生皆可完成答题任务。该系统可让教师轻松、清晰、准确地了解 学生的情况, 不仅提高了学生的课堂 “参与感”, 也可稳步推进教学进程, 促使教师及时 调整教学进度。

在医学物理学的教学过程中, 我们及时测验学生对知识的掌握程度。某一次, 我们出 了 10 道填空题、20 道选择题、10 道简答题。图 2 给出了部分截图, 我们可以看出, 浅绿 色的表示优秀, 绿色的表示正确, 而红色的表示错误。数据表明, 可以清楚地看到每一位 学生的得分情况和大多数学生失分的题目, 如 A25 这一道选择失分率较高。

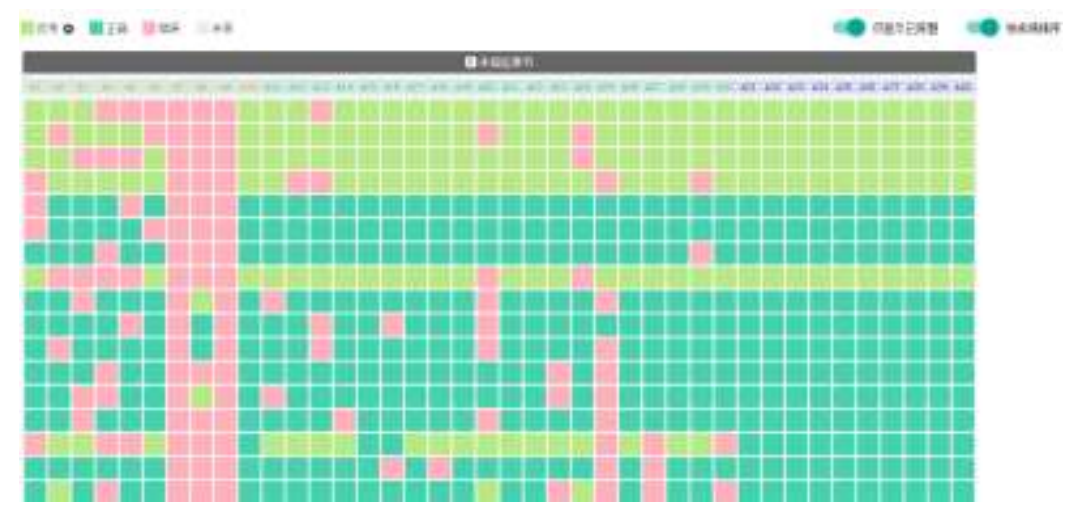

图 2 某测试正误分别情况图

在教学过程中, 也可以对某一题目进行详细的分析。例如, 某一次教学过程中, 我们 对医学物理学中的转动惯量概念和影响因素的教学, 为了测验和评价学生对知识的掌握程 度。我们出了如下测试题: 例 1、做定轴转动的刚体, 其转动惯量与下列哪些因素无关:
A. 刚体的质量;
B. 刚体所受合外力;
C. 刚体转轴的位置;
D. 刚体质量分布情 况;

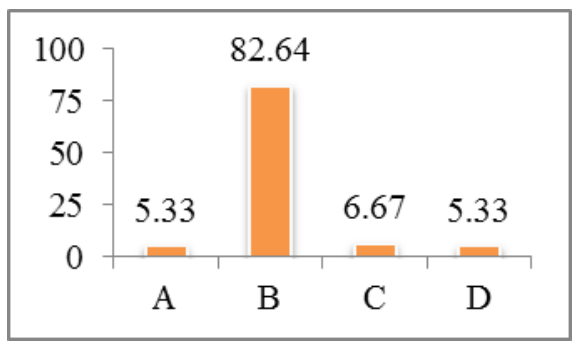

图 3 得分率及选项得分比率

\section{1 .3 多元的学生讨论互动}

积极有效的课堂互动是融洽师生关系, 活跃课堂气氛, 激发学生思维, 提高课堂质量 
和效率的重要手段。这可让教师充分了解每一个学生的动态, 了解他们的心理和学习活动 轨迹从而及时做出反馈, 比如个别谈话思想教育, 查漏补缺式学习辅导, 培优补差式辅导

\section{1.4 系统的云平台记录服务}

在 21 世纪, 信息化服务平台能让教学评价方式更有针对性、更精准。在 “微助教” 课堂教学体系里, 学生的出勤率、课堂答题参与率和正确率、课堂互动频率、虚拟论坛发 言、平时作业和小测验等, 都可以通过云平台记录下来, 有利于教师对学生学习的全过程 进行持续观察, 做出最后发展性的评价。

\section{2 “微助教” 在医学物理学教学中的思考}

（1）在医学物理学的教学理念中发挥微助教的优势: 随着时代的进步, 需要大家具 有开放学习的心态, 把握学生特点, 积极探索教学改革与研究, 并应用到实际的教学中去, 为促进学校教育事业发展发挥积极作用。

（2）在医学物理学课程的教学考勤中发挥微助教作用: “微助教” 能够带动课堂气氛 的活跃, 课堂由以前的 “老师点名” 向 “学生主动抢着签到” 转变, 不少学生为抢到了签 到 “第一名”而感到自豪。

(3) 在医学物理学的教学中, 发挥 “微助教” 优势, “医治”上课低头症：大学生如 果在 46 秒内不看手机, 就会产生焦虑情绪。通过手机终端操作取代传统纸笔的签到不仅 能提高学生课堂出勤率, 还能提高班级主人公意识, 制造班级归属感。学生几乎很少低头 睡觉或者打手游, 每一个知识片段之后, 学生将参与教学。

（4）在医学物理学的教学过程中, 正确认识和评价 “评分过严”带来的问题: 我们 的教学实践表明, “微助教” 在选择题的正确判断上是非常完美的, 不仅可以快捷地查看 答题人数、正确率等。但是, 对于填空题, 只要学生文字稍有出入, 系统的 “精准匹配功 能” 会判为错误, 答错了的题学生也无法进行修正, 这需要进一步优化。因此, 我们要对 这样的问题要进行二次分析, 以便正确的把握学生的学习情况。例如, 在几何光学的教学 中, 让学生熟记明视距离的数值。我们的测试题如下：例 2、在光照适宜的条件下, 不易 引起眼睛过度疲劳的距离约为 $\mathrm{cm}$, 称为明视距离。

我们可以清楚的看到, 75 人作答 $(96 \%)$, 正确率为 $73 \%$ 。

表 1 某填空题, 答案及人数分布

\begin{tabular}{lllllllllll}
\hline 答案 & 0 & 20 & 25 & 30 & 50 & 100 & $10 \mathrm{~cm}$ & $20 \sim 30$ & $25 \mathrm{~cm}$ & $30 \mathrm{~cm}$ \\
\hline 人数 & 1 & 1 & 55 & 5 & 1 & 1 & 1 & 1 & 13 & 1
\end{tabular}




\section{3 结语}

“微助教” 作为一种新型智慧课堂教学系统, “微助教” 课堂是对传统课堂的创新性 尝试和有效翻转。它不仅给予了教师高度的课堂 “控制感”, 也给予了学生高度的课堂 “参 与感”，给高等教育管理者也带来了 “安全感”。这种高度创新性的课堂教学使师生均都能 愉快的教与学, 是对信息化创新课堂的有效辅助。在医学物理学的教学实践中, 数据表明 提高了学生的出勤率、增加了学生参与课堂的积极性和热度、方便了教师掌握学生的学习 情况等等。我们相信, 在未来的信息化教育教学过程中, 我们教师会在教学中积极主动地 应用现代信息技术, 同时兼顾传统教学手段方法的综合应用。因此, 要崇尚创新的教学文 化氛围，进一步提高人才培养质量。

\section{4 致谢}

本研究得到 2017 年国家级大学生创新创业训练计划项目（批准号：201710929002)。

\section{Acknowledgement:}

We thank the supports from the2017 National Training Program of Innovation and Entrepreneurship for Undergraduates (Grant Nos. 201710929002).

\section{References}

[1] <http://news.163.com/16/1214/02/C87A0KM3000187VI.html>

[2] <http://mini.eastday.com/mobile/161010070454303.html>

[3] <http://news.cnr.cn/native/city/20161011/t20161011_523190310.shtml>

[4] <http://info.whu.edu.cn/info/1318/165104.htm>

[5] <http://xinwen.hbee.edu.cn/html/xwsp/2016-10/136245.html>

[6] <http://news.xinhuanet.com/local/2016-10/12/c_129319735.htm> 